\title{
Vestibular paroxysmia in vestibular neuritis: A case report
}

\author{
Michele Ori, Valeria Gambacorta, Giampietro Ricci, Mario Faralli \\ Department of Surgery and Biomedical Sciences, Section of Otorhinolaryngology, University of Perugia, Italy
}

\begin{abstract}
The term vestibular paroxysmia (VP) was introduced for the first time by Brandt and Dieterich in 1994. In 2016, the Barany Society formulated the International Classification of VP, focusing in particular on the number and duration of attacks, on the differential diagnosis and on the therapy. Ephaptic discharges in the proximal part of the eighth cranial nerve, which is covered by oligodendrocytes, are assumed to be the neural basis of VP. We report the first case in literature of an onset of symptoms and signs typical of VP in a young man following acute unilateral vestibular loss not combined with auditory symptoms. Indeed, the pathogenic mechanism affected only the vestibular nerve as confirmed by the presence of a stereotyped nystagmus pattern. The magnetic resonance imaging didn't reveal any specific cause therefore we suggest the possible role of a neuritis triggering an ephaptic discharge as the neural mechanism of VP.
\end{abstract}

\section{Introduction}

In 1975 Jannetta and colleagues described a neurovascular cross-compression in patients with hyperactive dysfunction symp-

Correspondence: Michele Ori, Clinica di Otorinolaringoiatria e Chirurgia Cervico-Facciale, Ospedale Santa Maria della Misericordia Sant'Andrea delle Fratte, 06129 Perugia (PG), Italy.

Tel. +39.075.5783236 - Fax: +39.075.5783574

E-mail: michele.ori357@gmail.com

Key words: Vestibular paroxysmia; vestibular neuritis; ephaptic discharge; young age.

Acknowledgments: the authors would like to thank Mrs. Deanna Anderlini for linguistic review.

Contributions: MO, VG, design of the work, data acquisition; GR, data acquisition, data analysis and interpretation; $\mathrm{MO}, \mathrm{VG}, \mathrm{GR}, \mathrm{MF}$, data analysis and interpretation, critical revise for important intellectual content, final approval of the version to be published; MF, work conception.

Conflict of interest: the authors declare no potential conflict of interest.

Received for publication: 8 February 2018.

Revision received: 22 March 2018.

Accepted for publication: 31 March 2018.

This work is licensed under a Creative Commons Attribution NonCommercial 4.0 License (CC BY-NC 4.0).

CC Copyright M. Ori et al., 2018

Licensee PAGEPress, Italy

Audiology Research 2018;8:206

doi:10.4081/audiores.2018.206 toms of the eighth cranial nerve. ${ }^{1}$ In 1984 the term disabling positional vertigo (DPV) was coined to describe a clinical heterogeneous syndrome of vertigo with highly variable characteristics in durations (from seconds to days), in features (spinning vertigo, light-headedness or gait instability without vertigo), and in accompanying symptoms. ${ }^{2}$ During the microvascular decompression of the nerve with the aim of reducing the symptoms of DPV, the authors found arteries and veins responsible for the compression, as assumed prior the treatment. ${ }^{3}$

The term vestibular paroxysmia (VP) was introduced for the first time by Brandt and Dieterich in $1994 .{ }^{4}$ Based on an observational study, the authors described a syndrome characterized by the following features: i) short attacks of spinning or non-spinning vertigo lasting from seconds to minutes; ii) attacks frequently dependent on a particular head position; iii) hyperacusis or tinnitus during the attacks or constantly present; and iv) neurophysiological evidence of auditory or vestibular deficit. For diagnosis at least three of the four criteria are required together with a good response to carbamazepine. ${ }^{4}$ In 2016 Barany Society formulated the diagnostic criteria for vestibular paroxysmia distinguishing definite VP from probable VP (Table 1). ${ }^{5}$ Major differential diagnoses are Tumarkin's otolithic crisis, vestibular migraine, benign paroxysmal positional vertigo, epileptic vestibular aura, paroxysmal brainstem attacks (in multiple sclerosis or after brainstem stroke), Minor's syndrome, perilymph fistula, transient ischemic attacks and panic attacks. ${ }^{5}$ We report the first case in literature of an onset of symptoms and signs typical of VP following acute unilateral vestibular loss (UVL) not combined with auditory symptoms. This highlights the possible role of neuritis as a cause of an ephaptic discharge in the assumed mechanism of VP.

\section{Case Report}

In June 2017, a 21 years old man was referred to our department with acute spontaneous vertigo, nausea/vomiting and unsteadiness at his awakening without tinnitus, ear fullness or hearing loss. The clinical ear-nose-throat examination on admission revealed a horizontal spontaneous nystagmus beating toward the left side (unaffected ear) with a rotational component visible with Frenzel glasses; the fixation partially inhibits the nystagmus. The nystagmus was persistent, one directional and pluri-positional during bedside examination, while paroxysmal positional nystagmus was not evoked. Postural imbalance with falls toward the right side (affected ear) was also present. The head impulse test (HIT) and caloric irrigation showed a deficit of the vestibulo-ocular reflex (VOR) on the right side. A deviation towards the right during the subjective visual vertical test indicated an utricular damage. An early biphasic complex (p1-n1) well recognizable for morphology was present and it appeared normal for amplitude and latency $(13-23 \mathrm{~m} / \mathrm{sec})$ in the recording of the cervical vestibular evoked myogenic potentials (cVEMPs) ipsilateral to the lesion, indicating that saccular function was preserved. Pure tone audiometry revealed normal hearing function bilaterally. All these clinical 
features led to the diagnosis of right superior nerve vestibular loss.

A few weeks later, the patient experienced an episode of benign paroxysmal position vertigo (BPPV) of the right posterior canal which was treated with modified Epley manoeuvres. The inferior vestibular pathway is known as responsible of the oculomotor response in the presence of fluctuating material in the posterior semicircular canal. As the pathway was not damaged, it was possible to make a diagnosis of Lindsay-Hemenway's syndrome.

Sometime later, in September 2017, he reported many brief attacks of spontaneous spinning vertigo (about 20 per day). A bedside examination was carried out during multiple attacks, and a stereotyped nystagmus was identified, both spontaneous (with and without fixation) and with the supine head roll test (PagniniMcClure manoeuvre) and Dix-Hallpike positional manoeuvre. The nystagmus had a horizontal component beating toward the affected side (right ear) of the same amplitude in each eye, a vertical downbeating component more evident in the right eye and a torsional component anticlockwise more evident in the left eye. The result of the two linear components, horizontal and vertical, was an oblique nystagmus downward and rightward more pronounced in the right eye (Figure 1).

The nystagmus was present in every position, generally with a total duration of 40-50 seconds. In order to exclude a pluricanal $\mathrm{BPPV}$, we performed repositioning manoeuvres during each consultation without effect neither on the nystagmus' features nor on the symptomatology. Hyperventilation-induced nystagmus with the same patterns was occasionally detected.

Brainstem auditory evoked responses were normal. Magnetic Resonance Imaging (MRI) findings excluded neuro-vascular cross-compression, the presence of a tumour in the area of the cerebello-pontine angle and other brainstem lesions. Therefore we decided to perform a video head impulse test (vHIT) which showed a right lowered VOR gain of 0.37 (Figure 2). The sequence of these new signs and symptoms, as well as the examinations' results, led us to the hypothesis of probable VP.

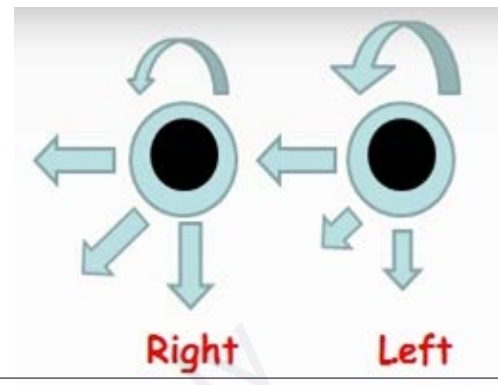

Figure 1. Schematic representation of the various components of the stereotyped nystagmus observed in both eyes during paroxysmal attacks of recurrent vertigo.

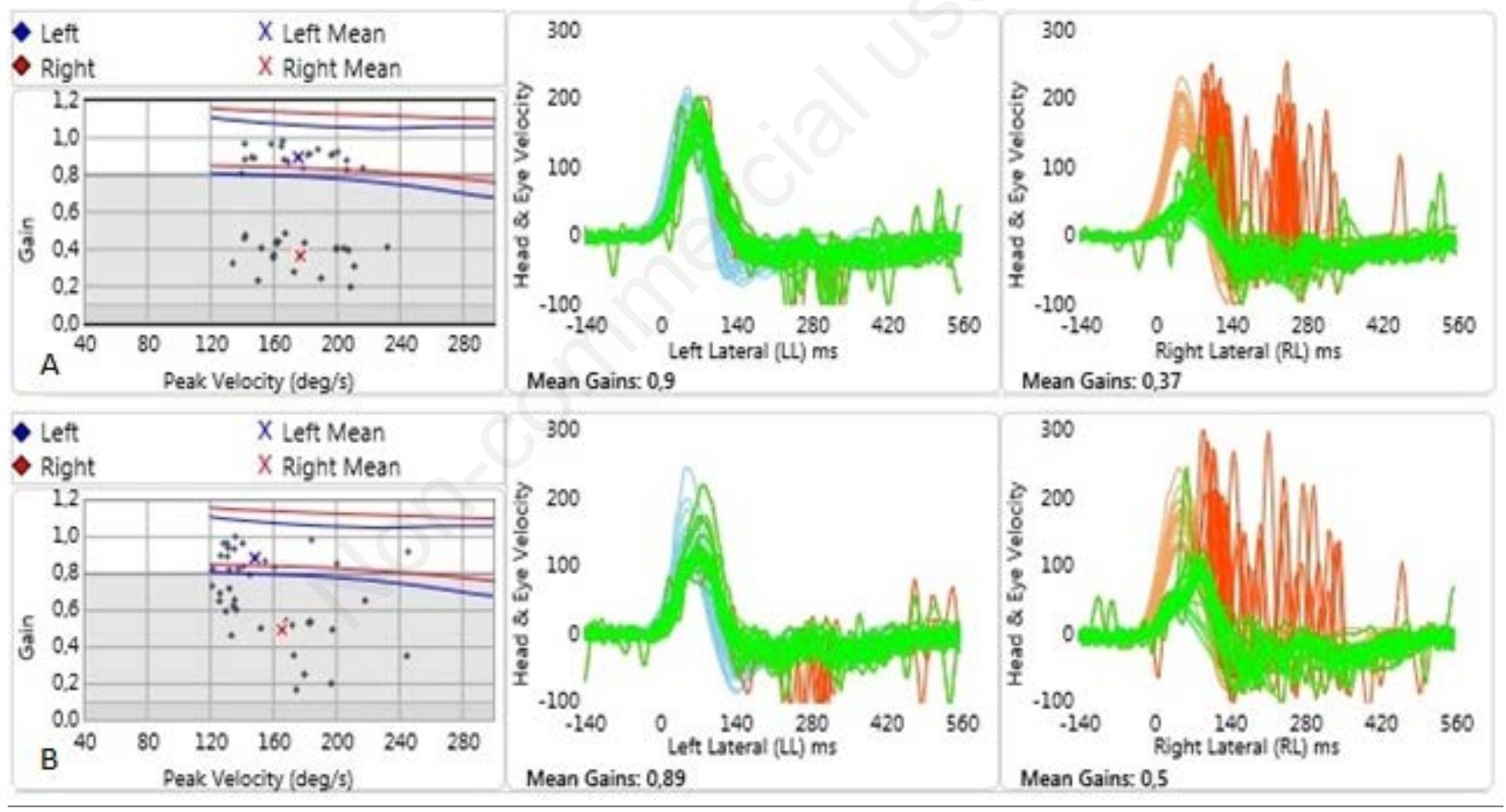

Figure 2. Video head impulse test at the time of the onset of signs and symptoms of vestibular paroxysmia (A) and during the follow up (B). Notice partial deafferentation of the right superior vestibular nerve at the beginning and progressive recovery of vestibulo-ocular reflex gain.

Table 1. Diagnostic criteria for vestibular paroxysmia.

\begin{tabular}{lll} 
Criteria & $\begin{array}{l}\text { Definite vestibular paroxysmia } \\
\text { (each point needs to be fulfilled) }\end{array}$ & $\begin{array}{l}\text { Probable vestibular paroxysmia } \\
\text { (each point needs to be fulfilled) }\end{array}$ \\
A & At least ten attacks of spontaneous spinning or non-spinning vertigo & $\begin{array}{l}\text { At least five attacks of spinning or non-spinning vertigo } \\
\text { Duration less than 5 minutes }\end{array}$ \\
\hline B & Duration less than 1 minute & Spontaneous occurrence or provoked by certain head-movements \\
D & Stereotyped phenomenology in a particular patient & Stereotyped phenomenology in a particular patient \\
\hline E & Response to a treatment with carbamazepine/oxcarbazepine & Not better accounted for by another diagnosis \\
\hline
\end{tabular}


The patient was examining weekly: bedside examination revealed the persistence of above-mentioned nystagmus, meanwhile he reported a step-by-step remission of spontaneous symptomatology. For this reason, no pharmacological therapy was prescribed. Written informed consent was obtained from the patient for publication of the report.

\section{Discussion}

The prevalence of VP is unknown since only small case series and a few single cases have been reported. ${ }^{4,6-10}$ In the case series the mean age of the patients varied from 48 (range $25-77$ years) ${ }^{9}$ to 51 years (range $25-67$ years). ${ }^{4}$ Although the age of our patient is not in those ranges, VP was also described in children with features similar to those in adults. ${ }^{10}$ It is assumed that VP is caused by neurovascular cross-compression at the root entry zone of the eight cranial nerve (N.VIII) which is covered by oligodendrocytes (central myelin). ${ }^{11}$ Apart from focal irritation by a blood vessel, other potential causes for nerve injury are compression by tumour/cyst, demyelination, trauma and unidentified causes. ${ }^{12,13}$

The most relevant features found in our case are: i) the exclusive involvement of the vestibular division of N.VIII confirmed by the vestibular assessment and the lack of a cochlear impairment; ii) the selective engagement of the superior vestibular nerve indicated by the onset of a secondary labyrintholithiasis of the posterior semicircular canal together with the presence of an early biphasic complex (p1-n1). The complex was well recognizable for morphology and it was normal for amplitude and latency (13-23 $\mathrm{m} / \mathrm{sec}$ ) in the recording of the cVEMPs ipsilateral to the lesion; iii) the occurrence of brief frequent episodes either in rest or induced by changes in head/body position matched the diagnosis criteria for probable VP; iv) the emergence of the same ocular pattern triggered by positional tests and hyperventilation, as a paroxysmal irritation of the superior vestibular nerve; $v$ ) the absence of other causes potentially able to lead to probable VP in the MRI study (focal irritation by a blood vessel, compression and demyelination).

In our case it is quite difficult to identify the real aetiology and physiopathology of the occurrence of a clinical entity that meets the diagnostic criteria for probable VP. On one hand, the sequence of events would lead to consider the possibility of an axonal damage secondary to a neuritis responsible for ephaptic discharges in the superior branch of the vestibular nerve, as some experimental studies showed. ${ }^{14,15}$ The absence of cochlear symptoms typically associated with probable VP supports also this hypothesis. On the other hand, the morphological and temporal characteristics of the stereotyped nystagmus would indicate a new distinct disease.

Regarding a new disease, it is conceivable that a causal factor with neural basis could be responsible of UVL and VP independently. The absence of an MRI documented neurovascular compression of the N.VIII in $5 \%$ of 32 patients with VP, ${ }^{9}$ and conversely the presence of compression in normal subjects, ${ }^{6}$ they support the idea of a new syndrome.

Vestibular neuritis is uncommon in young patients and no cases of secondary probable VP following UVL have neither been reported in older age groups. Therefore the age could represent a predisposing condition highlighting the viral aetiology of the affection mainly in young people as opposed to a vascular injury in the territory of the anterior vestibular artery, more frequent in the elderly. Moreover the young age would explain the not clearly quantifiable neural reaction, either myelin repair or neural plasticity and it would easily influence the clinical evolution of the illness. Finally, as corroborated by the VOR gain of 0.37 (Figure 2) at the time of the onset of the symptoms, an incomplete deaf- ferentation of the superior vestibular nerve appears to be an essential functional condition to account for ephaptic transmission, which would trigger paroxysmal attacks of recurrent vertigo.

An increase of the VOR gain during the follow-up (Figure 2) provides evidence of a functional recovery and it validates the clinical improvement. To date, the favourable progress and the lack of a definite etiologic mechanism convinced us not to treat this young patient with carbamazepine.

\section{Conclusions}

Although rare, VP secondary to vestibular neuritis should be considered in young patients, especially when auditory symptoms are missing because the pathogenic mechanism affects exclusively the vestibular nerve. This condition appears to have a good prognosis with spontaneous remission of symptoms and VOR recovery without carbamazepine's administration.

\section{References}

1. Jannetta PJ. Neurovascular cross-compression in patients with hyperactive dysfunction symptoms of eight cranial nerve. Surg Forum 1975;26:467-9.

2. Jannetta PJ, Møller MB, Møller AR. Disabling positional vertigo. N Engl J Med 1984;310:1700-5.

3. Møller MB, Møller AR, Jannetta PJ, Sekhar L. Diagnosis and surgical treatment of disabling positional vertigo. J Neurosurg 1986;64:21-8.

4. Brandt T, Dieterich M. Vestibular paroxysmia: vascular compression of the eighth nerve? Lancet 1994;343:798-9.

5. Strupp M, Lopez-Escamez JA, Kim JS, et al. Vestibular paroxysmia: diagnostic criteria. J Vestib Res 2016;26:409-15.

6. Best C, Gawehn J, Krämer HH, et al. MRI and neurophysiology in vestibular paroxysmia: contradiction and correlation. J Neurol Neurosurg Psychiatry 2013;84:1349-56.

7. Chang TP, Wu YC, Hsu YC. Vestibular paroxysmia associated with paroxysmal pulsatile tinnitus: a case report and review of the literature. Acta Neurol Taiwan 2013;22:72-5.

8. He LY, Dong WW, Huang W, et al. Clinical manifestations and imaging features of peripheral vestibular paroxysmia: a report of 7 cases. Zhonghua Yi Xue Za Zhi 2009;89:909-11.

9. Hüfner K, Barresi D, Glaser M, et al. Vestibular paroxysmia: diagnostic features and medical treatment. Neurology 2008;71:1006-14.

10. Lehnen N, Langhagen T, Heinen F, et al. Vestibular paroxysmia in children: a treatable cause of short vertigo attacks. Dev Med Child Neurol 2015;57:393-6.

11. De Ridder D, Møller A, Verlooy J, et al. Is the root entry/exit zone important in microvascular compression syndromes? Neurosurgery 2002;51:427-33.

12. Arbusow V, Strupp M, Dieterich M, et al. Alternating episodes of vestibular nerve excitation and failure. Neurology 1998;51:1480-3.

13. Lee SU, Jeong SH, Kim HJ, Kim JS. Cerebellopontine angle meningioma mimicking vestibular paroxysmia. J Neurol 2016;263:168-70.

14. Smith KJ, McDonald WI. Spontaneous and evoked electrical discharges from a central demyelinating lesion. J Neurol Sci 1982;55:39-47.

15. Smith KJ, McDonald WI. Spontaneous and mechanically evoked activity due to central demyelinating lesion. Nature 1980;286:154-5. 